\title{
Long Lasting Effects of Breastfeeding on Metabolism in Women with Prior Gestational Diabetes
}

\author{
Luca Mattei*, Antonietta Colatrella, Olimpia Bitterman, Paola Bianchi, Chiara Giuliani, \\ Giona Roma, Camilla Festa, Gianluca Merola, Vincenzo Toscano, Angela Napoli \\ Faculty of Medicine and Psychology, Sapienza University of Rome, Rome, Italy \\ Email: mluca982@hotmail.it
}

Received 7 August 2014; revised 5 September 2014; accepted 1 October 2014

Copyright (C) 2014 by authors and Scientific Research Publishing Inc.

This work is licensed under the Creative Commons Attribution International License (CC BY). http://creativecommons.org/licenses/by/4.0/

(c) (i) Open Access

\section{Abstract}

Background \& Aims: Breastfeeding improves glucose tolerance in the early postpartum period of women with prior gestational diabetes GDM, but it is unclear whether future risk of metabolic alterations, like type 2 diabetes, is reduced. The aim of this study was to investigate the effect of lactation, three years after pregnancy, on glucose and lipid metabolism in women with prior gestational diabetes. Materials \& Methods: A population of women with prior gestational diabetes (Carpenter and Coustan Criteria) was evaluated with comparison of results for "lactating" [BF] versus "nonlactating women" [non BF]. Breast feeding was defined [BF] if lasting? 4 weeks. In each woman a 75-g oral glucose tolerance test (OGTT) was performed to analyze the glucose tolerance, insulin sensitivity/resistance and b-cell function. Fasting serum was used to study their lipid profile (total cholesterol, high-density lipoprotein [HDL] cholesterol, low-density lipoprotein [LDL] cholesterol, and triglycerides), apolipoprotein B, apolipoprotein A1, homocysteine, fibrinogen, hsCRP, uric acid, microalbuminuria. Statistics: Paired and Un-paired t-test, Mann-Whitney and $\chi^{2}$ tests were used, as appropriate. Results: A total of 81 women were evaluated (62 [BF] and 19 [non $B F])$. Maternal age $(37.1 \pm 4.6$ vs $37.4 \pm 4.9$ years $)$, body mass index $\left(26.3 \pm 5.6 \mathrm{vs} 26.4 \pm 5.3 \mathrm{~kg} / \mathrm{m}^{2}\right)$, parity $(1.9 \pm 0.8 \mathrm{vs} 1.7 \pm 0.8)$ and length of follow-up $(32.2 \pm 20.2$ vs $32.1 \pm 20,0)$ were not different between the two groups. No effect was visible on glucose tolerance, HOMA-IR and other b-cell function indexes as well as hs-CRP (not significantly lower in non BF), uric acid, total cholesterol, HDL and LDL cholesterol. Levels of significance were only reached for "HOMA-IS" [BF] $1.0 \pm 0.7 \mathrm{vs}$ [non $\mathrm{BF}] 0.6 \pm 0.4, \mathrm{p}=0.04$ ) and triglycerides $[\mathrm{BF}] 83.8 \pm 46.7 \mathrm{vs}[\mathrm{non} \mathrm{BF}] 123.2 \pm 94.0 \mathrm{mg} / \mathrm{dl}, \mathrm{p}=0.02$ ). Conclusions: Breastfeeding does not improve the glucose tolerance of our women with prior GDM three years after delivery, even though lower levels of triglycerides and improved insulin sensitivity are still visible.

\footnotetext{
${ }^{*}$ Corresponding author.
} 


\section{Keywords}

\section{Breastfeeding, Gestational Diabetes, Metabolism}

\section{Background}

Gestational diabetes mellitus (GDM) is common and transient glucose intolerance, first diagnosed during pregnancy and often resolving at delivery. However, women with GDM have an increased risk for postpartum abnormalities in insulin secretion and insulin action, as well as type 2 diabetes [1].

In these women, a deficit in insulin secretion/action may be observed even if post-partum glucose tolerance is normal, as well as different levels of insulin resistance [2].

Therefore, a "healthy" lifestyle and/or the presence of other risk factors after pregnancy can influence the chance of developing diabetes later in life [3].

Breastfeeding is recommended by many health agencies as the best method to feed infants for at least one year after birth, because of its multiple immediate and long-term benefits for both child and mother (infectious diseases, obesity, immune-related diseases; breast cancer) [4].

Breastfeeding confers health benefits to women with a history of GDM in terms of glucose tolerance in the early postpartum period [5], but it's unclear whether future risk of type 2 diabetes is reduced [6] [7].

A 2001 study found that within a matched population of women with a history of GDM, three months of breastfeeding was associated to improved pancreatic beta-cell function, but not to any significant difference in glucose tolerance, adipose tissue mass, or adipose distribution [8].

The primary aim of this study was to investigate the effect of breastfeeding on glucose metabolism as well as on beta cell function in women with prior GDM, three years after pregnancy.

The secondary aim was to evaluate in these women lipid and inflammatory profile and the prevalence of metabolic syndrome (M.S.).

\section{Patients and Methods}

Women were successively recruited from January 2007 to December 2009 in our 'Diabetes in Pregnancy” outpatient's office, at S. Andrea Hospital of "Sapienza” University, Rome.

A cohort of 81 women, three years after a pregnancy complicated by GDM (diagnosed through Carpenter \& Coustan Criteria) [9], with negative anti GAD antibodies at diagnosis, was evaluated according to their breastfeeding habits. Also patients with just one altered value in the OGTT [defined OAV ("one abnormal value")] had been considered [10].

None of the women was treated with medications and/or substances potentially conditioning metabolism at the moment of follow up.

Arbitrarily, we defined breastfeeding only if lactation duration was longer than four weeks.

Other parameters evaluated at follow up visit were: age, follow up length (measured as months after delivery), BMI $\left(\mathrm{kg} / \mathrm{m}^{2}\right)$, waist circumference $(\mathrm{cm})$, systolic and diastolic blood pressure, conventionally measured $(\mathrm{mmHg})$ and familiarity for type 2 diabetes.

Laboratory parameters studied were: total, LDL and HDL-cholesterol, triglycerides, Apo lipoprotein B, Apo lipoprotein A1, homocysteine, fibrinogen, hs-CRP, uric acid, TSH, micro albuminuria.

At follow up visit, in the morning, after an overnight fasting period of at least eight hours, all patients performed a 75 g OGTT for glycaemia and insulin (samples at 0', 30' 60', 90' and 120'). All blood samples were analysed in the same laboratory in our university hospital. Plasma glucose levels were determined with a Beckman Glucose Analyzer 2 by glucose oxidase method, and plasma insulin concentration was measured by radioimmunoassay.

Women were defined normal, IFG (impaired fasting glucose), IGT (impaired glucose tolerance) or diabetic, through basal glucose measurements \pm OGTT according to ADA diagnostic criteria [11].

Insulin sensitivity and secretion indexes were obtained using plasma glucose and insulin values in the OGTT; insulin sensitivity was estimated using HOMA and HOMA calculator [12] and ISI as proposed by Matsuda et al. [13]. To evaluate $\beta$-cell secretion, we considered an insulinogenic index expressed as Ins30'/Glic30', a surro- 
gate of the first phase of insulin secretion, and the areas under glucose and insulin curves (AUC), calculated by a trapezoidal method [14]. Disposition index was used as $\beta$-cell function index [15].

Metabolic syndrome and hypertension were defined by ATP III 2004 criteria [16].

All the biochemical tests were performed in Sant' Andrea university hospital laboratory, in Rome.

\subsection{Statistic}

We used Paired \& Un-Paired $t$-test, Mann-Whitney \& $\chi^{2}$ tests. Data concerning glucose profile are expected to follow a normal distribution and are expressed in terms of their mean and standard deviation.

\subsection{Informed Consent}

According to our usual practice, a written informed consent was obtained by all the women attending our outpatient's office at the first visit in pregnancy, later confirmed after delivery and at the moment of the last clinical and metabolic evaluation.

\section{Results}

\subsection{General Characteristics}

A cohort of 81 women was followed up about three years after delivery (32.2 \pm 20.2 months after delivery). According to our definition of breastfeeding habit, 62 women were classified as breastfeeding only [BF] and 19 as not-breastfeeding [non-BF]. At the follow up visit none of the women was still lactating.

The two groups were similar in terms of follow up length, age, parity, number of children, smoking habit, type 2 diabetes familiarity in first-degree relatives. On average, women breastfed for $25.5 \pm 19.2$ weeks (range 4 - 96 weeks). Regarding anthropometric parameters, there were no differences in BMI and waist circumference (Table 1).

\subsection{Glycaemic Homeostasis}

According to the number of glucose alteration in the OGTT performed in pregnancy, BF group included 46 GDM and $16 \mathrm{OAV}$, while non-BF group included $14 \mathrm{GDM}$ and $5 \mathrm{OAV}$. Moreover, 63\% BF and 78\% non-BF women $\left(\chi^{2}, \mathrm{~ns}\right)$ required insulin treatment.

At follow up, no difference was found in glucose and insulin levels at each time point in the OGTT. Among non-BF women, 13 (68.4\%) were normal, 4 (21.0\%) were IFG, 1 (5.3\%) was diabetic and 1 was IGT/IFG (5.3\%); among BF women 44 (71.0\%) were normal, 5 was IFG (8.0\%), 6 were IGT (9.7\%), and 7 were diabetic $(11.3 \%)\left(\chi^{2}, \mathrm{~ns}\right)$. These two groups were similar in terms of insulin resistance indexes, except for HOMA-IS (BF $1.0 \pm 0.7$ vs non-BF $0.6 \pm 0.4, \mathrm{p}=0.04)$. No differences were found in insulinogenic index and disposition index (Table 2).

\section{Table 1. General characteristics.}

\begin{tabular}{cccc}
\hline & BF $\mathbf{n}=\mathbf{6 2 / 8 1}$ & non-BF $\mathbf{~}=\mathbf{1 9 / 8 1}$ & p \\
\hline Follow up length (months) & $32.2 \pm 20.2$ & $32.1 \pm 20.0$ & 0.86 \\
Age (y) & $37.1 \pm 4.6$ & $37.2 \pm 5.1$ & 0.89 \\
Parity (n) & $2.3 \pm 1.1$ & $2.1 \pm 1.0$ & 0.39 \\
Number of child (n) & $1.9 \pm 0.89$ & $1.68 \pm 0.88$ & 0.31 \\
Smoking habit (\%) & 15 & 25 & 0.85 \\
DMT2 familiarity (\%) & 53.3 & 47.1 & 0.64 \\
BMI (kg/m ${ }^{2}$ ) & $26.3 \pm 5.6$ & $26.4 \pm 5.3$ & 0.86 \\
\hline Waist circumference (cm) & $87.5 \pm 10.4$ & $83.5 \pm 10.3$ & 0.20 \\
\hline
\end{tabular}


Table 2. Glycaemic homeostasis.

\begin{tabular}{|c|c|c|c|}
\hline & $B F n=62 / 81$ & non-BF $n=19 / 81$ & $\mathbf{p}$ \\
\hline OGTT 0 & $90.1 \pm 16.4$ & $92.2 \pm 8.9$ & 0.61 \\
\hline OGTT 30 & $142.5 \pm 34.3$ & $140.1 \pm 40.1$ & 0.82 \\
\hline OGTT 60 & $140.7 \pm 42.4$ & $124.0 \pm 33.0$ & 0.16 \\
\hline OGTT 90 & $113.6 \pm 36.8$ & $107.0 \pm 32.1$ & 0.54 \\
\hline OGTT 120 & $101.5 \pm 28.1$ & $101.2 \pm 26.9$ & 0.97 \\
\hline $\begin{array}{l}\text { OGTT alteration (\%) } \\
\text { DM/IFG/IGT }\left(\mathrm{n}^{\circ}\right)\end{array}$ & $\begin{array}{c}29 \\
7 \text { DM, } 5 \text { IFG, } 6 \text { IGT }\end{array}$ & $\begin{array}{c}31.6 \\
1 \mathrm{DM}, 4 \mathrm{IFG}, 1 \mathrm{IFG} / \mathrm{IGT}\end{array}$ & 0.34 \\
\hline Homa-IR & $2.2 \pm 3.9$ & $2.7 \pm 2.7$ & 0.64 \\
\hline ISI & $8.1 \pm 4.2$ & $6.8 \pm 3.6$ & 0.34 \\
\hline Homa-IS & $1.0 \pm 0.7$ & $0.6 \pm 0.4$ & 0.04 \\
\hline Insulinogenic index & $14.1 \pm 14.0$ & $11.7 \pm 29.9$ & 0.68 \\
\hline AUCIRI/AUCBG & $0.3 \pm 0.2$ & $0.4 \pm 0.2$ & 0.80 \\
\hline Disposition index & $2.5 \pm 1.08$ & $1.96 \pm 0.62$ & 0.78 \\
\hline Cholesterol (mg/dl) & $191.9 \pm 37.7$ & $203.4 \pm 40$ & 0.31 \\
\hline HDL (mg/dl) & $55.9 \pm 14.7$ & $55.7 \pm 15.4$ & 0.95 \\
\hline LDL (mg/dl) & $116.3 \pm 40.7$ & $120.3 \pm 29.6$ & 0.76 \\
\hline Triglycerides (mg/dl) & $83.8 \pm 46.7$ & $123.2 \pm 94.0$ & 0.03 Mann-Whitney \\
\hline ApoA1 (mg/dl) & $147.6 \pm 22.9$ & $150 \pm 37.9$ & 0.79 \\
\hline ApoB (mg/dl) & $93.13 \pm 25.8$ & $106.8 \pm 23.6$ & 0.13 \\
\hline АроB/ApoA1 & $0.65 \pm 0.23$ & $0.73 \pm 0.16$ & 0.31 \\
\hline hs-CRP (mg/L) & $0.5 \pm 0.7$ & $2.3 \pm 6.2$ & 0.08 \\
\hline Homocysteine $(\mu \mathrm{moli} / \mathrm{L})$ & $8.6 \pm 3.2$ & $7.9 \pm 2.3$ & 0.52 \\
\hline Systolic (mmHg) & $112.17 \pm 15.7$ & $110 \pm 11.9$ & 0.66 \\
\hline Diastolic (mmHg) & $71.6 \pm 10.6$ & $72.6 \pm 9.2$ & 0.73 \\
\hline MS/ATPIII (\%) & 50.0 & 27.7 & 0.67 \\
\hline
\end{tabular}

\subsection{Lipidic Profile}

There were no differences in total cholesterol, HDL and LDL levels between the two groups. Significant lower triglycerides values were observed in BF women (BF $83.8 \pm 46.7$ vs non-BF $123.2 \pm 94.0 \mathrm{mg} / \mathrm{dl}$; p = 0.03, Mann Whitney) (Table 2).

\subsection{Inflammatory Markers}

There were no differences in inflammatory markers: hs-CRP, homocysteine. We did not find any difference in ApoA1 and ApoB values and ApoB/ApoA1 ratio (Table 2).

\subsection{Blood Pressure and Metabolic Syndrome}

We did not found significant differences either in systolic or diastolic blood pressure values.

No difference was observed in prevalence of MS (Table 2). 


\section{Discussion}

According to our results, breastfeeding has no long term impact on glucose tolerance in women with prior GDM. Three years after delivery, OGTT glycaemic levels were similar at each time point in the two groups, as well as insulin resistance and secretion indexes. These results are partially explained by the fact that more than $60 \%$ of the studied population needed insulin treatment. An improvement in metabolic profile is observed in breastfeeding women with mild GDM [5].

However, HOMA-IS index still suggests a protective role of breastfeeding on insulin sensitivity; while the other insulin sensitivity indexes show a similar trend, without reaching the level of significance.

Many studies investigated the role of breast-feeding early after delivery. McManus et al. demonstrated a greater beta-cell compensation to insulin resistance, associated to lower OGTT glycaemic levels, in women with recent GDM still BF, three months after delivery. The same authors did not find any significant difference in adipose tissue mass or distribution through CT [8]. These results are likely to be due to a higher glucose consumption for milk production through a non insulin-related pathway. Therefore, a reduction in glucose stimulation on beta-cells could potentially prevent progression towards type 2 diabetes, as observed by Kjos, who showed a lower diabetes prevalence in BF women (4.2\% BF vs 9.4\% non-BF). Moreover, despite diabetes was more frequent in women who needed insulin therapy during pregnancy, breast feeding was still a protective factor at follow up, so that in insulin treated group $12.6 \%$ of "BF" resulted affected from diabetes compared to $22 \%$ of “non-BF” [5].

Recently, the "Atlantic DIP Study” confirmed the protective role of breastfeeding 12 weeks after delivery in a cohort of women from five Irish regions, 300 with previous GDM and 220 with normal glucose tolerance in pregnancy. In the OGTT performed after pregnancy, 19\% of the women with previous GDM vs $2.7 \%$ of controls had an impaired glucose tolerance, with 8.2\% reduction of "persistent hyperglycaemia” in BF vs non-BF. Risk factors were non-European ethnic group, type diabetes family history, higher BMI and insulin therapy in pregnancy, while breastfeeding had a protective role [17].

There are few follow-up studies investigating if the protective effect of lactation on late development of insulin resistance and insulin secretion defects persists after weaning. Diniz did not observe any association between breastfeeding duration and insulin sensitivity in 67 women from health population evaluated 12 and 18 months after delivery [18]. Chouinard-Castonguay showed that a total breastfeeding period of at least ten months leads to improved glucose tolerance as well as improved insulin sensitivity and insulin secretion [19].

The "Nurses Health Study", a prospective observational cohort study on 83,585 parous women, and the “Nurses Health Study II”, a retrospective observational cohort study on 73,418 parous women, showed that long lasting breastfeeding, particularly if exclusive, is associated to a fifteen percent decrease of type 2 diabetes risk, for each additional year. However, the same studies failed to find any protective effect of breastfeeding on diabetes risk, in women with a history of gestational diabetes [20].

Two studies on Latin American women with previous GDM, showed that diabetes diagnosis were halved in breastfeeding patient 4 - 12 weeks after delivery, but not after 11 - 26 months [21] [22].

Ziegler and colleagues prospectively investigated the impact of breastfeeding on diabetes outcome in women with prior GDM up to 19 years after delivery and observed a $>40 \%$ long term risk reduction. Diabetes risk was related to treatment received during pregnancy (insulin vs diet), BMI, and presence/absence of islet autoantibodies. Among islet autoantibody-negative women, breastfeeding was associated to a median period to diabetes diagnosis of 12.3 years compared to 2.3 years in women who did not breastfeed. The lowest postpartum diabetes risk was observed in women who breastfed for 3 months [23].

Recently, a systematic review shows a 9\% reduction in relative risk for developing T2DM for each 12-month increase in lifetime duration of breastfeeding and this inverse association appears to be independent of other important risk factors for type 2 diabetes, including BMI, smoking, alcohol, physical activity, education, income, parity and family history of diabetes [24].

In our study, breastfeeding was not correlated either to type 2 diabetes or any alteration of glucose tolerance (IGT and/or IFG and/or Diabetes), as well as to BMI and the other investigated risk factors.

Several studies demonstrated that lipid panel can be modified by breastfeeding [5]. Kjos et al. found higher HDL cholesterol levels in BF women with previous GDM, 4 - 12 weeks after delivery. In our study lower triglycerides levels were found in "BF women", with no difference in total, HDL and LDL cholesterol.

Gunderson et al. noted an inverse association between breastfeeding and metabolic syndrome incidence in women with previous GDM; in particular, the incidence rate of metabolic syndrome was 6 fold lower when 
women breastfed at least nine months [25]. Our results do not confirm the protective role of breastfeeding on metabolic syndrome, by contrast of what observed by the "CARDIA Study", 20 years after delivery.

Two-thirds of our population is breastfed exclusively. Recently, Matias et al. demonstrated that maternal obesity, insulin treatment, and suboptimal in-hospital breastfeeding were essential risk factors for delayed lactogenesis onset [26].

Finally, as ours is a retrospective study, we do not have data about some of the many variables affecting metabolic syndrome risk, such as physical activity and food habits, during follow-up. However, all the women had received general recommendations about lifestyle to prevent metabolic diseases.

\section{Conclusions}

Breastfeeding protective role on metabolic profile in women with previous GDM is still evident in a long-term follow up study.

All the available resources should be used already in pregnancy to implement education programs [27].

\section{References}

[1] Bellamy, L., Casas, J.P., Hingorani, A.D. and Williams, D. (2009) Type 2 Diabetes Mellitus after Gestational Diabetes: A Systematic Review and Meta-Analysis. Lancet, 373, 1773-1779. http://dx.doi.org/10.1016/S0140-6736(09)60731-5

[2] Retnakaran, R., Qi, Y., Sermer, M., Connelly, P.W. and Hanley, A.J.G. and Zinman, B. (2010) $\beta$-Cell Function Declines within the First Year Postpartum in Women with Recent Glucose Intolerance in Pregnancy. Diabetes Care, 33, 1798-1804. http://dx.doi.org/10.2337/dc10-0351

[3] Schack-Nielsen, L. and Michaelsen, K.F. (2006) Breast Feeding and Future Health. Current Opinion in Clinical Nutrition and Metabolic Care, 9, 289-296. http://dx.doi.org/10.1097/01.mco.0000222114.84159.79

[4] American Dietetic Association (1997) Position of the American Dietetic Association: Promotion of Breastfeeding. Journal of the American Dietetic Association, 97, 662-666. http://dx.doi.org/10.1016/S0002-8223(97)00167-3

[5] Kjos, S.L., Henry, O., Lee, R.M., Buchanan, T.A. and Mishell Jr., D.R. (1993) The Effect of Lactation on Glucose and Lipid Metabolism in Women with Recent Gestational Diabetes. Obstetrics Gynecology, 82, 451-455.

[6] Gunderson, E.P. (2007) Breastfeeding after Gestational Diabetes Pregnancy. Diabetes Care, 30, S161-S168. http://dx.doi.org/10.2337/dc07-s210

[7] Aune, D., Norat, T., Romundstad, P. and Vatten, L.J. (2014) Breastfeeding and the Maternal Risk of Type 2 Diabetes: A Systematic Review and Dose-Response Meta-Analysis of Cohort Studies. Nutrition, Metabolism Cardiovascular Diseases, 24, 107-115. http://dx.doi.org/10.1016/j.numecd.2013.10.028

[8] McManus, R.M., Cunningham, I., Watson, A., Harker, L. and Finegood, D.T. (2001) Beta-Cell Function and Visceral Fat in Lactating Women with a History of Gestational Diabetes. Metabolism—Clinical and Experimental, 50, 715-719. http://dx.doi.org/10.1053/meta.2001.23304

[9] Carpenter, M.W. and Coustan, D.R. (1982) Criteria for Screening Test for Gestational Diabetes. American Journal of Obstetrics Gynecology, 144, 768-773.

[10] Langer, O., Brustman, L., Anyaegbunam, A. and Mazze, R. (1987) The Significance of One Abnormal Glucose Tolerance Test Value on Adverse Outcome in Pregnancy. American Journal of Obstetrics Gynecology, 157, 758-763. http://dx.doi.org/10.1016/S0002-9378(87)80045-5

[11] American Diabetes Association (2014) Diagnosis and Classification of Diabetes Mellitus. Diabetes Care, 37, S81-S90. http://dx.doi.org/10.2337/dc14-S081

[12] Matthews, D.R., Hosker, J.P., Rudenski, A.S., Naylor, B.A., Treacher, D.F. and Turner, R.C. (1985) Homeostasis Model Assessment: Insulin Resistance and $\beta$-Cell Function from Fasting Plasma Glucose and Insulin Concentrations in Man. Diabetologia, 28, 412-419. http://dx.doi.org/10.1007/BF00280883

[13] Matsuda, M. and DeFronzo, R. (1999) Insulin Sensitivity Indices Obtained from Oral Glucose Tolerance Testing. Comparison with the Euglycemic Insulin Clamp. Diabetes Care, 22, 1462-1470. http://dx.doi.org/10.2337/diacare.22.9.1462

[14] Stumvoll, M., Mitralou, A., Pimenta, W., Jenssen, T., Yki-Jarvinen, H., Van Haeften, T., Renn, W. and Gerich, J. (2000) Use of the Oral Glucose Tolerance Test to Assess Insulin Release and Insulin Sensitivity. Diabetes Care, 23, $295-301$. http://dx.doi.org/10.2337/diacare.23.3.295

[15] Utzschneider, K.M., Prigeon, R.L., Faulenbach, M.V., Tong, J., Carr, D.B., Boyko, E.J., Leonetti, D.L., McNeely, M.J., Fujimoto, W.Y. and Kahn, S.E. (2009) Oral Disposition Index Predicts the Development of Future Diabetes Above and Beyond Fasting and 2-h Glucose Levels. Diabetes Care, 32, 335-341. http://dx.doi.org/10.2337/dc08-1478 
[16] Grundy, S.M., Brewer Jr., H.B., Cleeman, J.I., Smith Jr., S.C. and Lenfant, C. (2004) Definition of Metabolic Syndrome: Report of the National Heart, Lung, and Blood Institute/American Heart Association Conference on Scientific Issues Related to Definition. Circulation, 109, 433-438. http://dx.doi.org/10.1161/01.CIR.0000111245.75752.C6

[17] O’Reilly, M.W., Avalos, G., Dennedy, M.C., O’Sullivan, E.P. and Dunne, F. (2011) Atlantic DIP: High Prevalence of Abnormal Glucose Tolerance Postpartum Is Reduced by Breast-Feeding in Women with Prior Gestational Diabetes Mellitus. European Journal of Endocrinology, 165, 953-959. http://dx.doi.org/10.1530/EJE-11-0663

[18] Diniz, J.M. and Da Costa, T.H. (2004) Independent of Body Adiposity, Breastfeeding Has a Protective Effect on Glucose Metabolism in Young Adult Women. British Journal of Nutrition, 92, 905-912. http://dx.doi.org/10.1079/BJN20041288

[19] Chouinard-Castonguay, S., Weisnagel, S.J., Tchernof, A. and Robitaille, J. (2013) Relationship between Lactation Duration and Insulin and Glucose Response among Women with Prior Gestational Diabetes. European Journal of Endocrinology, 168, 515-523. http://dx.doi.org/10.1530/EJE-12-0939

[20] Stuebe, A.M., Rich-Edwards, J.W., Willett, W.C., Manson, J.E. and Michels, K.B. (2005) Duration of Lactation and Incidence of Type 2 Diabetes. JAMA, 294, 2601-2610. http://dx.doi.org/10.1001/jama.294.20.2601

[21] Kjos, S.L., Peters, R.K., Xiang, A., Henry, O.A., Montoro, M. and Buchanan, T.A. (1995) Predicting Future Diabetes in Latino Women with Gestational Diabetes: Utility of Early Postpartum Glucose Tolerance Testing. Diabetes, 44, 586591. http://dx.doi.org/10.2337/diab.44.5.586

[22] Buchanan, T.A., Xiang, A.H., Kjos, S.L., Trigo, E., Lee, W.P. and Peters, R.K. (1999) Antepartum Predictors of the Development of Type 2 Diabetes in Latino Women 11-26 Months after Pregnancies Complicated by Gestational Diabetes. Diabetes, 48, 2430-2436. http://dx.doi.org/10.2337/diabetes.48.12.2430

[23] Ziegler, A.G., Wallner, M., Kaiser, I., Rossbauer, M., Harsunen, M.H., Lachmann, L., Maier, J., Winkler, C. and Hummel, S. (2012) Long-Term Protective Effect of Lactation on the Development of Type 2 Diabetes in Women with Recent Gestational Diabetes Mellitus. Diabetes, 61, 3167-3171. http://dx.doi.org/10.2337/db12-0393

[24] Aune, D., Norat, T., Romundstad, P. and Vatten, L.J. (2014) Breastfeeding and the Maternal Risk of Type 2 Diabetes: A Systematic Review and Dose Response Meta-Analysis of Cohort Studies. Nutrition, Metabolism and Cardiovascular Diseases, 24, 107-115. http://dx.doi.org/10.1016/j.numecd.2013.10.028

[25] Gunderson, E.P., Jacobs Jr., D.R., Chiang, V., Lewis, C.E., Feng, J., Quesenberry Jr., C.P. and Sidney, S. (2010) Duration of Lactation and Incidence of the Metabolic Syndrome in Women of Reproductive Age According to Gestational Diabetes Mellitus Status: A 20-Year Prospective Study in CARDIA (Coronary Artery Risk Development in Young Adults). Diabetes, 59, 495-504. http://dx.doi.org/10.2337/db09-1197

[26] Matias, S.L., Dewey, K.G., Quesenberry Jr., C.P. and Gunderson, E.P. (2014) Maternal Prepregnancy Obesity and Insulin Treatment during Pregnancy Are Independently Associated with Delayed Lactogenesis in Women with Recent Gestational Diabetes Mellitus. American Journal of Clinical Nutrition, 99, 115-121. http://dx.doi.org/10.3945/ajcn.113.073049

[27] World Health Organization (2013) Health Topics, Nutrition Topics, 10 Facts on Breastfeeding. http://www.who.int/features/factfiles/breastfeeding/facts/en/index.html 
Scientific Research Publishing (SCIRP) is one of the largest Open Access journal publishers. It is currently publishing more than 200 open access, online, peer-reviewed journals covering a wide range of academic disciplines. SCIRP serves the worldwide academic communities and contributes to the progress and application of science with its publication.

Other selected journals from SCIRP are listed as below. Submit your manuscript to us via either submit@scirp.org or Online Submission Portal.
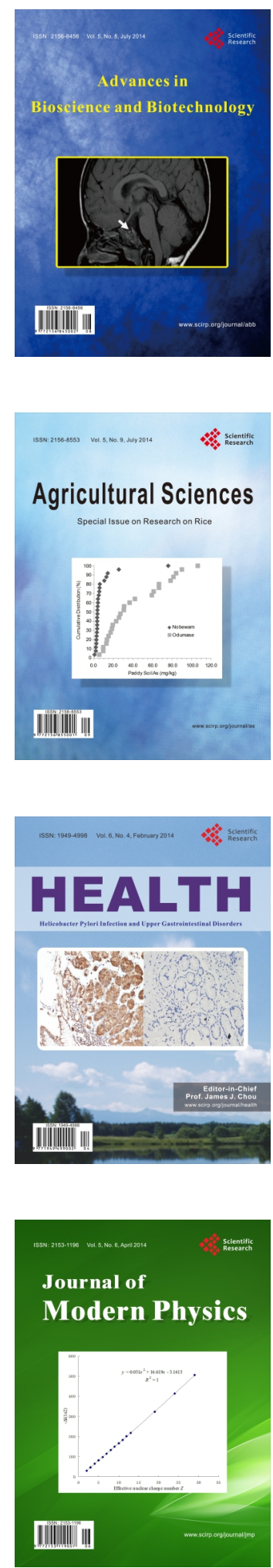
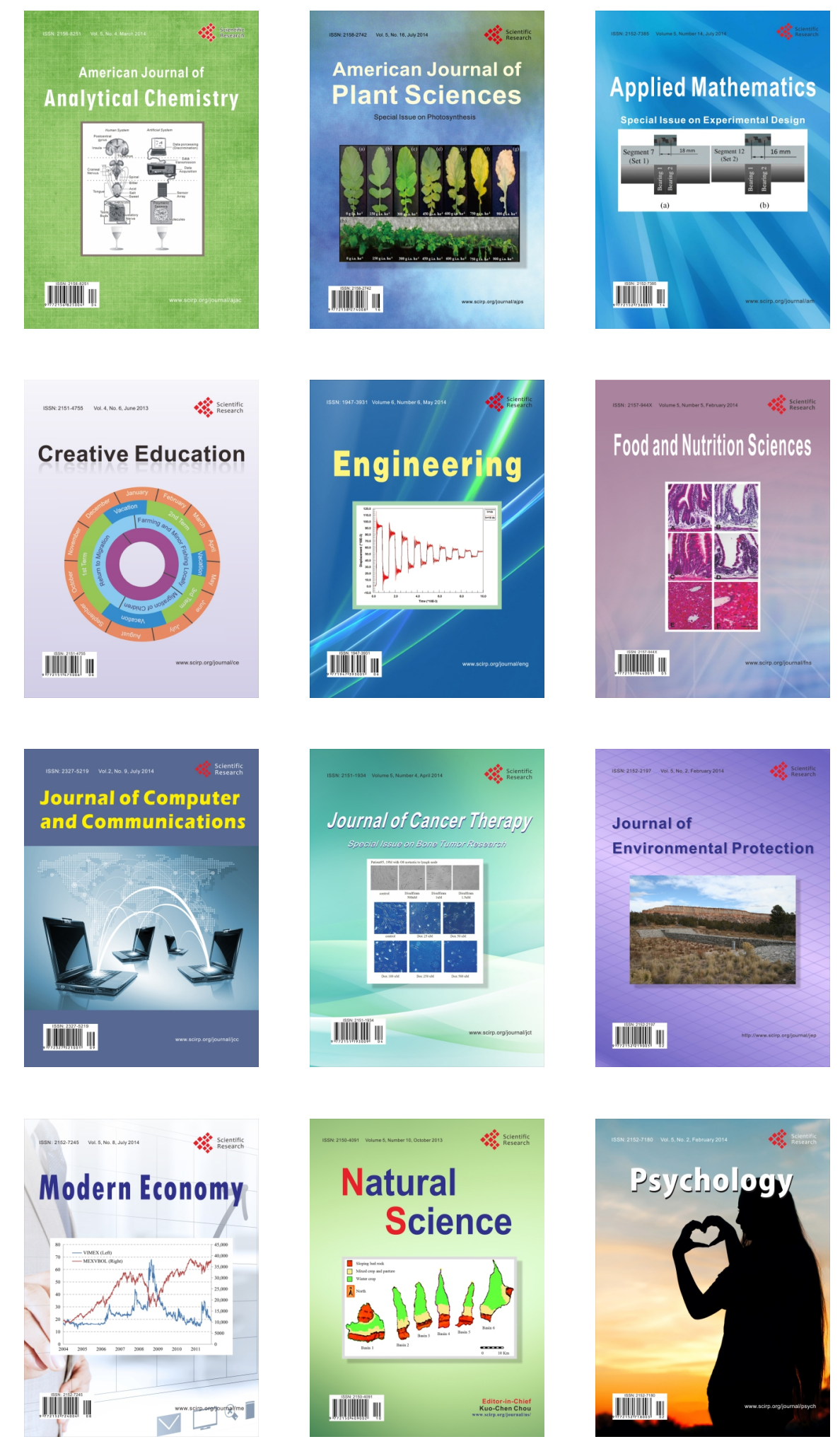